INPLASY

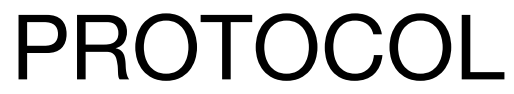

To cite: Zhu et al. Efficacy and safety of anti-CD19 chimeric antigen receptor-T cells immunotherapy in patients with relapsed or refractory large B-cell lymphoma: a systematic review and metaanalysis. Inplasy protocol 202190045. doi:

10.37766/inplasy2021.9.0045

Received: 15 September 2021

Published: 15 September 2021

Corresponding author:

Zhitao Ying

yingzhitao001@163.com

Author Affiliation:

Department of Lymphoma, Peking University Cancer

Hospital \& Institute.

Support: JW Therapeutics.

Review Stage at time of this submission: The review has not yet started.

Conflicts of interest:

None declared.

\section{Efficacy and safety of anti-CD19 chimeric antigen receptor-T cells immunotherapy in patients with relapsed or refractory large B-cell lymphoma: a systematic review and meta-analysis}

Zhu, J1; Song, Y2; Ying, Z3.

Review question / Objective: We aimed to conduct a metaanalysis to evaluate the efficacy and safety of anti-CD19 chimeric antigen receptor-T cells immunotherapy in patients with relapsed or refractory large B-cell lymphomas.

Condition being studied: Large B-cell lymphoma, as the most common non-Hodgkin lymphoma (NHL), acoounts for $30 \%$ of NHL with the rising incidence. The first line chemotherapy regimen could alleviate symptoms in $60 \%-70 \%$ LBCL patients, while $30 \%-40 \%$ of LBCL patients would be difficult to treat to relapse post the first response, with poor prognosis. Chimeric antigen receptor $T$ cells(CAR-T) is the latest developed cellimmunotherapy in recent years and demonstrates remarkable achievements in lymphoblastic leukemia with its unique advantages.

INPLASY registration number: This protocol was registered with the International Platform of Registered Systematic Review and Meta-Analysis Protocols (INPLASY) on 15 September 2021 and was last updated on 15 September 2021 (registration number INPLASY202190045).

\section{INTRODUCTION}

Review question / Objective: We aimed to conduct a meta-analysis to evaluate the efficacy and safety of anti-CD19 chimeric antigen receptor-T cells immunotherapy in patients with relapsed or refractory large B-cell lymphomas.

Condition being studied: Large B-cell lymphoma, as the most common nonHodgkin lymphoma (NHL), acoounts for $30 \%$ of NHL with the rising incidence. The 
first line chemotherapy regimen could alleviate symptoms in $60 \%-70 \%$ LBCL patients, while $30 \%-40 \%$ of LBCL patients would be difficult to treat to relapse post the first response, with poor prognosis. Chimeric antigen receptor T cells(CAR-T) is the latest developed cell-immunotherapy in recent years and demonstrates remarkable achievements in lymphoblastic leukemia with its unique advantages.

\section{METHODS}

Search strategy: We started systematic search from databases: PubMed, Embase and Cochrane library from available papers in literature from inception to Aug. 2021 for potentially eligible studies.

Participant or population: Patients with advanced, relapsed, or chemotherapyrefractory large B cell lymphoma were included.

Intervention: (Chimeric Antigen Receptor T Cells) OR (CAR-T[Title/Abstract]) AND CD19[Title/Abstract].

\section{Comparator: Not applicable.}

Study designs to be included: Clinical trials and observational studies in full-text or conference abstract, case report is excluded.

Eligibility criteria: 1) Patients with advanced, relapsed, or chemotherapyrefractory large $B$ cell lymphoma were included; 2) Cases were more than ten; 3) CD 19- CAR T cells immunotherapy; 4) Fulltext in English.

Information sources: Databases: PubMed, Embase and Cochrane library from available papers in literature.

Main outcome(s): Efficacy outcome: objective response rate (ORR), progression free survival (PFS), overall survival (OS), complete response (CR), partial response (PR), cell amplification, cytokines. Safety outcome: Cytokine release syndrome (CRS), neurological event, decreased immunoglobulin.
Quality assessment / Risk of bias analysis: MINORS for non-randomized clinical trials, NOS criteria for case-control and cohort studies. MINORS, for single arm studies.

Strategy of data synthesis: Efficacy and Safety: The pooled odds ratios (event rate) estimate of ORR, CR, PR and safety outcomes with $95 \%$ confidence intervals (CI) were obtained using the randomeffects model.

Subgroup analysis: 1) Commercial product / CAR-T from Laboratory. 2) Different combination therapy. Other analysis. CRS: cumulative meta-analysis by time (start date of trials).

Sensitivity analysis: We would find the source of heterogeneity through sensitivity analysis by eliminating literature one by one. The sensitivity analysis of the primary outcome will be provided.

Language: English.

Country(ies) involved: China.

Keywords: Large B-cell Iymphoma, Chimeric antigen receptor $T$ cells, immunotherapy, CAR-T cell therapy, metaanalysis.

Contributions of each author:

Author 1 - Jun Zhu.

Email: zhujun@csco.org.cn

Author 2 - Yuqin Song.

Email: songyuqin622@163.com

Author 3 - Zhitao Ying.

Email: yingzhitao001@163.com 